\title{
The neural, evolutionary, developmental, and bodily basis of metaphor
}

\author{
Jay A. Seitz ${ }^{\mathrm{a}, \mathrm{b}, *}$ \\ ${ }^{a}$ Department of Political Science and Psychology, AC-4D06, York College/City University of New York (CUNY), \\ Jamaica, NY 11451, USA \\ ${ }^{\mathrm{b}}$ New School University, USA
}

\begin{abstract}
We propose that there are four fundamental kinds of metaphor that are uniquely mapped onto specific brain "networks" and present preliterate (i.e., evolutionary, including before the appearance of written language in the historical record), prelinguistic (i.e., developmental, before the appearance of speech in human development), and extralinguistic (i.e., neuropsychological, cognitive) evidence supportive of this view. We contend that these basic metaphors are largely nonconceptual and entail (a) perceptual-perceptual, (b) cross-modal, (c) movement-movement, and (d) perceptual-affective mappings that, at least, in the initial stages of processing may operate largely outside of conscious awareness. In opposition to our basic metaphor theory $\left(B_{m} T\right)$, the standard theory $\left(S_{m} T\right)$ maintains that metaphor is a conceptual mapping from some base domain to some target domain and/or represents class-inclusion (categorical) assertions. The $\mathrm{S}_{\mathrm{m}} \mathrm{T}$ captures aspects of secondary or conceptual metaphoric relations but not primary or basic metaphoric relations in our view. We believe our theory $\left(\mathrm{B}_{\mathrm{m}} \mathrm{T}\right)$ explains more about how people actually recognize or create metaphoric associations across disparate domains of experience partly because they are "pre-wired" to make these links.
\end{abstract}

(C) 2005 Elsevier Ltd. All rights reserved.

Keywords: Metaphor; Creativity; Embodied cognition; Brain and mind; Unconscious

\footnotetext{
*Corresponding author. Department of Political Science and Psychology, AC-4D06, York College/City University of New York (CUNY), Jamaica, NY 11451, USA. Tel.: + 17182622691.

E-mail address: seitz@york.cuny.edu.

URL: http://www.york.cuny.edu/ seitz/bio.html.
} 


\section{Introduction}

We propose that there are four fundamental kinds of metaphor that are uniquely mapped onto specific brain "networks" and present preliterate (i.e., evolutionary, including before the appearance of written language in the historical record), prelinguistic (i.e., developmental, before the appearance of speech in human development), and extralinguistic (i.e., neuropsychological, cognitive) evidence supportive of this view. We contend that these basic metaphors are largely nonconceptual and entail (a) perceptualperceptual, (b) cross-modal (synesthetic), (c) movement-movement, and (d) perceptualaffective mappings that, at least, in the initial stages of processing may operate outside of conscious awareness. That is, they are informationally encapsulated (Fodor, 1983), "prewired" (Marcus, 2004), and probably universal across human populations (Brown, 1991, $1999,2000)$. In opposition to our basic metaphor theory $\left(B_{m} T\right)$, the standard theory $\left(S_{m} T\right)$ maintains that metaphor is a conceptual mapping from some base domain to some target domain (e.g., Gentner, Holyoak \& Kokinov, 2001; Lakoff \& Johnson, 1980) and/or represents class-inclusion (categorical) assertions (e.g., Glucksberg, 2003).

For instance, Gentner and colleagues (Gentner et al., 2001; Gentner \& Markman, 1997) argue that similarity, including metaphoric similarity as well as analogy, involves a process of "structure alignment" - a one-to-one correspondence between mapped elements and "systematicity" - a matching of connected systems of relations. Metaphors, however, map both systems of relations (e.g., atomic structure is like a solar system) and attributes of objects (e.g., "His hand was like a vise") depending on the type of the metaphor. This differentiation of similarity, however, conflates analogy with metaphor and $\mathrm{B}_{\mathrm{m}} \mathrm{T}$ theory suggests that metaphor is a separate species, at least for the metaphorical relations subsumed by it. On the other hand, Glucksberg and colleagues (Glucksberg, 2003; Glucksberg \& Keysar, 1993) contend that metaphors are more properly thought of as "implicit class-inclusion relations." For instance, the metaphorical statement, "Cigarettes are time bombs," is a class-inclusion assertion in which the terms, "cigarettes" and "time bombs" are absorbed under the superordinate category, "time bombs." Nevertheless, what is left out of both of these accounts is how people actually go about selecting which relations, which attributes or which terms to attend to in the first place.

While Glucksberg and colleagues maintain that metaphoric statements are apprehended as ordinary language - "quickly and automatically"-it would appear that metaphor comprehension probably occurs in conscious awareness because it involves, among other things, ongoing decisions about how to respond to mutually constituted social interactions (e.g., Speaker A: "Cigarettes are time bombs." Speaker B: "Yes, I stopped smoking several years ago"); although the authors do not confront this thorny aspect of metaphoric language - that it often serves some conscious social purpose. Indeed, Gentner and colleagues indicate that analogy and metaphor involve more abstract conceptual relations and presumably entail some conscious mechanisms.

People make analogical and metaphoric inferences based on higher-order connecting relations (p. 239).

Similar accounts are found elsewhere in the literature and are widespread, i.e., metaphor involves the mapping of conceptual domains at high levels of abstraction and entails some conscious mechanisms (e.g., Fauconnier \& Turner, 1998, 2002; Lakoff \& Johnson, 1980). Lakoff (1993), however, suggests that conceptual metaphor is largely unconscious and 
automatic like ordinary language and this largely unconscious substrate of our conceptual system "contains thousands of conventional metaphorical mappings" (p. 245). We criticize this view below under "Alternative Theories of Early Metaphor." In any event, the nonconscious mechanisms operating outside awareness are notoriously difficult to pin down (Allen \& Reber, 1998).

To be sure, consciousness deals with situations that require novel and nonstereotypical responses. Nonconscious mechanisms, however, function to deal with stereotypical forms of behavior conditioned by evolution for immediate and efficient use (Koch, 2004; Searle, 2005). We propose that the kinds of basic metaphoric relations in $B_{m} T$ are largely nonconscious and automatic: Notwithstanding individual differences, we might easily perceive a plate of spaghetti as a collection of worms or a stop sign as a popsicle (perceptual-perceptual metaphors), a spinning top as a ballerina (movement-movement metaphor), a front of a car as smiling (perceptual-affective metaphor) or music as sad (cross-modal metaphor; see Seitz, 2001a, 2005). That is, they involve extensive automaticity including implicit perception, memory, and thought; they are fast and independent (Marcus, 2004); they are engaged by specific environmental stimuli; and they are largely innate (Kihlstrom, 1987, 2002; see below). Cytowic (2002a, b) makes a related case for synesthesia arguing that that it is automatic, involuntary, and inborn.

$\mathrm{S}_{\mathrm{m}} \mathrm{T}$ thus captures aspects of secondary or conceptual metaphoric relations but not primary or basic metaphoric relations in our view. We think our theory $B_{m} T$ explains more about how people actually create or recognize metaphoric associations across disparate domains of experience partly because they are "pre-wired" to make these links. That is, we propose that humans have specialized neural subsystems for metaphoric understanding and production that are fine-tuned by experience and continuously modulated by underlying genetic mechanisms (Marcus, 2004).

The fundamental ability to link disparate sensory, perceptual, enactive, and affective domains can be described as an inter-, poly-, or multisensory ability or supra-, trans-, hetero- or multimodular capacity (Calvert, 2001, Seitz, 1997). That is, these basic metaphoric relations $\left(\mathrm{B}_{\mathrm{m}} \mathrm{T}\right)$ rely on central sensory, perceptual, and affective mechanisms that relate percepts and affects across dissimilar psychological domains based on relations within and between brain areas or "networks" (see below). For instance, biological constraints in the maturation of the intersensory areas in the mid- and frontal areas of the brain underlie synesthetic metaphor or the ability to link disparate sensory modalities (Marks, 1996; Marks \& Bornstein, 1987; Marks, Hammeal, \& Bornstein, 1987; Ramachandran \& Hubbard, 2001a, b).

Similarly, we have shown that the ability to link the physical and psychological domains ("psychological-physical metaphor;" Cicone, Gardner, \& Winner, 1981) is based on the earlier ability to attribute affective properties to inanimate objects or what we have called "physiognomic metaphor" (Seitz, 1998b; Seitz \& Beilin, 1987). For instance, whereas synesthetic metaphor is largely biological constrained, appears to derive from an inborn sense of similarity or comparison, and relies less on learning and development - that is to say, is largely intuitive (see below) - psychological-physical metaphor violates category boundaries based on learned conceptual relations (whether from informal experience or formal learning) and the probable rejection (whether conscious or not) of cultural and linguistic conventions. This is, at least, true of novel metaphors if not conventional ones (Gentner et al., 2001) as Quinn (1991) has argued: Metaphors are typically chosen by speakers because they are already part of existing cultural understandings. 
Nevertheless, the ability to relate the perceptual and affective domains originates in the biological capacity to impute affective properties to inanimate and animate objects (Seitz $\&$ Beilin, 1987). Autistic children who lack this capacity are unable to attribute affective properties to social or nonsocial objects or use or apprehend metaphoric language (BaronCohen, Leslie, \& Frith, 1985; Leslie \& Frith, 1988; Seitz, 1996)—notwithstanding Kanner's (1946) earlier but incorrect use of the term "metaphoric language" in autistic children (see Seitz, 1996) - as are brain damaged adults with alexithymia (Brothers, 1989) and adults with forms of prosopagnosia in which there is damage to anterior regions of area V4 in the inferior temporal lobe that underlie both affective recognition and expression in the face (Zeki, 1999).

\section{Intuition and metaphor}

\subsection{The role of intuition in the basic and standard theory}

$\mathrm{B}_{\mathrm{m}} \mathrm{T}$ posits that these four fundamental metaphors (i.e., perceptual-perceptual, crossmodal, movement-movement, and perceptual-affective relations) operate outside of awareness and manifest extensive automaticity in the initial stages of processing (Kihlstrom, 2002). That is, they are engaged by specific environmental stimuli (e.g., shape, size, texture, pitch, rhythm, rate, intensity, and spatial location) and the stimulus may not be consciously registered in its initial encounter. For instance, young children experience the fronts of cars as having affective expressions (Seitz \& Beilin, 1987) or experience sounds as being associated with colors or textures (Seitz, 1998a, b) without being consciously aware that they are doing so. This experience or "feeling of knowing" is very similar to the phenomenon of intuition and with the cognate view that creative thought occurs in four stages: Incubation, insight, confirmation, and verification (Wallas, 1926). Intuition has been defined as reaching conclusions based on nonconscious processes of reasoning (Gregory, 1998). Metaphoric intuition would operate both during the "incubation" stage in which unconscious ideas begin to coalesce and during the "insight" stage in which new ideas begin to emerge into consciousness. This is not to say that much of our everyday perceptual and cognitive apparatus (e.g., parsing sentences, accommodating the eyes to an object in the distance) does not occur outside of awareness; this is a truism.

Bowers and colleagues (Bowers, Regehr, Balthazard, \& Parker, 1990) have investigated the way intuition works during each of these two stages. In what they call the initial guiding stage, there is an unconscious and implicit perception of coherence that activates pertinent semantic networks in a graded and cumulative manner. "Hunches" and "hypotheses" emerge from underlying unconscious cognitive processes, i.e., in the course of a "preanalytic" or "automatic" stage of inquiry. Basic metaphorical processes of the kind described in $\mathrm{B}_{\mathrm{m}} \mathrm{T}$, in both children and adults, would be operative during this guiding or "incubation" stage of unconscious perception. Priming, an example of perceptual learning, has many features of this initial guiding stage of intuition. Squire and Kandel (1998) have argued that (a) priming is a form of nondeclarative memory, (b) that is has a brain basis (areas V1 and V2, see below), and (c) occurs in brain areas thought to be nonconscious (Koch, 2004).

In the second or "insight" stage, more advanced conceptual metaphor and analogical reasoning abilities would emerge. Bowers et al. (1990) reason that such an "integrative 
stage" would require the ability to engage in both extensive unconscious and conscious memory search (e.g., by way of spreading activation within a neuronal network) for corresponding attributes or relations for any two (or more) domains under comparison. Everyday experience, formal schooling, linguistic conventions, and social and cultural traditions, moreover, would shape these ongoing cognitive mechanisms (Seitz, 1999b, 2003a,b). Conceptual metaphor and more advanced analogical operations would be operative during this "integrative" stage of comparison.

In the following section, we look at the developmental emergence of these basic metaphoric abilities in infants and young children.

\section{Developmental evidence}

\subsection{Perceptual metaphor}

It has been amply documented that, early on, very young children (i.e., the beginning of the third year of life) are able to exploit the perceptual features of shape, color, texture, and size in making metaphorical associations in renaming single objects or pictures (e.g., Seitz, 1997, 1998a, b; Winner, McCarthy, \& Gardner, 1980; Winner, McCarthy, Kleinman, \& Gardner, 1979). For instance, a preschool child may refer to a plate of spaghetti as a "bunch of worms" or a vehicular caution sign as a "lemon ice cream cone." They can note these correspondences both in language as well as in match-to-sample tasks and similar nonverbal formats in which they must chose an analogous object from an array of pictorial or nonverbal choices (e.g., Kogan, Connor, Gross, \& Fava, 1980; Seitz, 1997, 1998b).

\subsection{Enactive metaphor}

Correspondingly, young children can make use of movement and motion informationexploit enactive components of metaphor - in both categorizing moving objects and in making metaphorical associations (Dent, 1984; Seitz, 1999a, 2000a, c, 2002). For example, a preschool child can note the metaphorical similarity between a spinning top and a dancing ballerina in a match-to-sample or similar nonverbal (e.g., filmed) format (Dent, 1984). Likewise, infants (18-24 months) can note the enactive similarity between a paper crayon cover that has slipped off and the putting on of an article of clothing, "I am putting your clothes on crayon" (Winner et al., 1980).

\subsection{Cross-modal (synesthetic) metaphor}

In a similar manner, infants and young children can perceive likeness in different sensory modalities (e.g., Gardner, 1974; Marks et al., 1987; Seitz, 1997, 1998a, b; Spelke, 1981). An instance of such a synesthetic or cross-modal association would be a 3-month-old infant that dishabituates to the synchrony between a toy monkey and its complementary sound (Spelke, 1981), a 3-year old that applies the polar adjectives "light/dark" to an object (e.g., sandpaper) felt while blindfolded (Gardner, 1974) or a preschooler who indicates that red is a warm or hot color (Seitz, 1997). Indeed, the distinguished art historian, Ernest Gombrich (1963), has long observed that such "natural metaphors"-bright sounds or cool colors - arise spontaneously in perception because they are part of our innate constitution. 


\subsection{Physiognomic metaphor}

During the very early preschool years rudimentary physiognomic experiences - the attribution of affective properties to visually perceived objects as well as other sensory experiences (e.g., identifying the front grille of an automobile as a "smiling face" or a piece of music as "cheerful") — gain prominence (Seitz, 1992, 1997, 1998a, b; Seitz \& Beilin, 1987). In one large study, child and adult responses to physiognomically suggestive visual metaphor were examined in photographs (Seitz \& Beilin, 1987). Preschoolers, 3 and 4 years of age, normal and high IQ children, 6 and 8 years of age, and adults were shown 10 photographs of cars, rocks, plants, and other inanimate objects and their responses categorized by type of metaphor (i.e., physiognomic characteristics, physical similarity and orientation, and intertextual, gestural-facial, and allegorization responses). Preschool and 6-year-old children demonstrated significant levels of physiognomic responding, although high IQ, older children, and adults showed even higher levels of physiognomic responding presumably as a result of more advanced narrative abilities. All groups displayed high consistency in physiognomic object responses across photographs and were equally good in categorizing a photograph using orientational metaphors. It therefore appears that physiognomic metaphor is a robust phenomenon across the lifespan, originating in the very early childhood years, at least as early as the second half of the third year of life (Seitz, 2001b; Seitz \& Beilin, 1987).

\subsection{Two distinct developmental phases}

Indeed, we have shown in several studies that the developmental emergence of basic $\left(\mathrm{B}_{\mathrm{m}} \mathrm{T}\right)$ and conceptual metaphor $\left(\mathrm{S}_{\mathrm{m}} \mathrm{T}\right)$ occurs in two distinct developmental phases that parallel the emergence of the guiding and integrative stages of intuition. In a test of this two-stage theory, 240 children 3- to 7-years-of-age were given a series of metaphor comprehension tasks in both the visual and verbal modality (Seitz, 1997, 1998a; Seitz \& Beilin, 1987). In the initial stage, children 4-years-of-age and younger exhibited a proclivity to violate category boundaries that were innately constrained (i.e., perceptual, synesthetic, and physiognomic metaphor). In the later stage, however, older children evidenced an inclination to violate category boundaries that relied on learning (e.g., formal schooling and experience) and/or rejection of cultural, social, and linguistic conventions. These included psychological-physical metaphors (e.g., "Bill was as cool as a cucumber") and conceptual or abstract metaphors (e.g., "The bird chirped like a violin"). As a result, it was suggested that adult creativity necessarily draws on both early metaphor that is largely innately constrained $\left(\mathrm{B}_{\mathrm{m}} \mathrm{T}\right)$ as well as later developing secondary or conceptual metaphor $\left(\mathrm{S}_{\mathrm{m}} \mathrm{T}\right)$ that relies on informal and formal learning and (in novel metaphor) the rejection of linguistic, social, and cultural conventions. Conceptual metaphor, moreover, would be based on something akin to Lakoff and Johnson's notion of experiential similarity or cooccurrence (Lakoff \& Johnson, 1980). They suggest that metaphorical concepts are assembled from either similarity encountered in the environment (e.g., noting the similarity between life and a gambling game as in "Where is he when the chips are down?") or correlations between domains as a result of their co-occurrence (e.g., experiencing "more" and "up" together as in "My income rose last year").

Nonetheless, evolutionary evidence indicates that the foregoing ontogeny of metaphor in the infant and young child has a clear parallel in the evolution of metaphoric abilities in 
early primate culture as well as early and late-appearing hominid groups. Evolutionary evidence is important because it provides a lens on the adaptive advantages such abilities in humans would serve and for what biological purposes they may have originally evolved (Sober \& Wilson, 1997).

\section{Evolutionary theories}

\subsection{Perceptual and synesthetic metaphor}

Merlin Donald (1991) has suggested that in the evolution of primates, metaphoric or "integrative" thought originated in early primate "episodic culture" associated with the initial appearance of perceptual metaphor including cross-modal associations. For what reason would these abilities have evolved in early primates? Early primate cognition would have necessitated the ability to engage in complex event discrimination ("episodic cognition") and to react to complex patterns of stimuli based on earlier experiences ("episodic memory") and such abilities would have been associated with both object and cross-modal perception. Comparable abilities, of course, are found in human infants (e.g., Lewkowicz, 1994, Spelke, 1981; Wagner, Winner, Cicchetti, \& Gardner, 1981) and highlight the central role of neoteny in human development. Neoteny is the slowing down of development in the ontogeny of descendents such that a mental or physical ability in juveniles of ancestral forms is retained in adults (Gould, 1977). In this case, episodic cognition in humans is a cognitive legacy (i.e., an archaic cognitive ability) inherited from earlier primates that proved useful to later evolving human primates in understanding conspecifics, their interactions, and events they initiated or encountered in the natural and social environment (i.e., "episodic culture," Bownds, 1999).

\subsection{Enactive metaphor}

Subsequently, enactive metaphor emerged in early hominid "mimetic" cultures, according to Donald (1991). He maintains that early hominid cognition would have required the ability to model behavior of conspecifics through vocal prosody, eye movement and gaze, facial expression, manual signs and gestures, rhythm, postural attitudes, and whole-body movements. These early abilities would have combined primate event perception with a complex map of the body tied to self-initiated actions. That is, early hominids would have developed the ability to kinesthetically model perceptual events in the environment as well as the ability to recall those events, both putatively mediated by recently discovered "mirror neurons" in the human cortex (see below). These capabilities would have evolved, according to Donald (1991), to facilitate toolmaking, fire and tool use, acclimation to seasonal changes in ecology and climate, participation in seasonal group hunting, and these capabilities would have most likely promoted social bonding through such actions as primitive pantomimic rituals. Not surprisingly, pretend action metaphors appear in the speech and gestures of very young children (Winner, 1988).

\subsection{Nonverbal metaphor}

More advanced metaphoric operations appeared, however, as human "mythic" cultures evolved approximately 250,000 years ago, precipitating language abilities and integrative 
mythical thought. Such abilities would have undergirded linguistic and complex gestural uses of metaphor as well as simple analogies (see Seitz, 1998b, for a review of the role of metaphor in gesture as well as the emergence of rudimentary analogy in children). Such metaphoric thought in early human cultures - primarily iconics (e.g., movements which depict a bodily action), beats (i.e., movements which accent vocal speech), and metaphoric gestures (e.g., movements which depict a spatial relation) - would have enabled the integration of events across individual episodes and facilitated the ability to extract thematic content paving the way for symbolic languages and discourse, i.e., paradigmatic (logical) and syntagmatic (narrative) thought. Iconic gestures, moreover, have been reported to emerge in human infants before 13 months of age (McNeill, 1992) and have been also reported in modern apes (Tomasello \& Call, 1997).

Nonetheless, more complex analogical reasoning including use of visual metaphor and other rhetorical devices were the outcome of modern human "theoretic" (i.e., symbolic) cultures that arose during the Upper Paleolithic period, 60,000 or so years ago (see also MacWhinney, 2002). Under this evolutionary scenario, visual thinking emerged and extended metaphoric and iconic gesture to the verbal realm in which linguistic and other symbols were imputed to visual experiences (e.g., "The road was a rocket of sunlight"). In this sense, verbal metaphor (and the conveyance of metaphoric relations through other symbols) is secondary to action and perception in which metaphoric abilities originate. In young children, visual metaphor emerges very early in child development (Seitz, 1998b; Winner, 1988). Donald (1991), thus, provides an evolutionary and biological basis for emergence of perceptual, enactive, and synesthetic forms of metaphor in primate and hominid phylogeny.

\subsection{Physiognomic metaphor}

With regard to physiognomic forms of metaphor, Stephen Mithen (1996) contends that early metaphoric abilities arose in human evolution during the Middle/Upper Paleolithic period approximately 40,000 to 60,000 years ago due to increasing "cognitive fluidity" that allowed language to be metaphorically extended from primarily social to nonsocial uses. He contends that anthropomorphic (i.e., inanimate objects given human characteristics) and totemic thought (i.e., the notion that humans are descended from various animals) likely precursors of physiognomic metaphor-arose late in human evolution and had selective advantages including the ability to improve the predictive power and reliability of securing food and other essential material goods from animal and plant prey.

The emergence of rock art during this same period is instructive. Three types of signs are found in early rock art: (a) pictograms, i.e., mythograms or representational figures of real or imaginary objects that consist of zoomorphic (animal-like) or anthropomorphic (human-like) features; (b) ideograms or synthetic and repetitive signs (e.g., tree-shapes, phallic and vulvar signs, and groups of lines and dots); and (c) psychograms or sharp graphic strokes that express human perceptual experiences. Anati (1994) suggests that these drawn and carved figures have metaphorical significance. For example, a particular sign may stand in place of individuals or tribal groups (hence, functioning metonymically) or suggest an interplay between male and female attributes or qualities (hence, functionally metaphorically). Studies of cave art have come to similar conclusions suggesting that the origins of Paleolithic art originate in the body and in a complex interplay between human and animal signs (Vialou, 1996). We suggest that such prehistoric signs have a strong 
affinity with physiognomic metaphor in which a percept is infused with affective tone (see Seitz, 2001b, for an explanation of the allure of such percepts in modern projective testing). Physiognomic metaphor, moreover, emerges very early in human development (Seitz \& Beilin, 1987). Mithen (1996), Anati (1994), and Vialou (1996), therefore, provide an evolutionary basis for the emergence of physiognomic forms of metaphor in later-evolving hominid populations. We turn now to the comparative evidence.

\section{Comparative evidence}

Comparative studies across species indicate that the core abilities underlying metaphor - such as the ability to form mental representations of perceived objects (i.e., to categorize and form concepts of objects), integrate sensory and affective experiences of those objects (i.e., cross-modal transfer), and form relational concepts - may be shared with other birds and mammals (Hauser, 2000; Pepperberg, 1999; Tomasello \& Call, 1997). For instance, in all investigated primate species there is evidence for cross-modal transfer between the visual and haptic modalities. Additionally, chimpanzees have demonstrated the ability to transfer between the visual and gustatory modalities, between the visual and auditory modalities, as well as across some classes of symbolic stimuli. Bonobos demonstrate the latter ability. Bushbabies (Prosimians) are able to transfer learned responses from the visual to the auditory modality. Chimpanzees and other apes and monkeys (i.e., rhesus macaques, squirrel, and capuchin monkeys) can form relational categories including the understanding of identity, oddity, and same-difference problems in match-to-sample like tasks. Chimpanzees are also capable of constructing multiple groupings of objects in a spontaneous manner and even engage in insight in solving some kinds of physical problems (Tomasello \& Call, 1997). Psittacines (i.e., parrots) demonstrate Piagetian stage 6 object permanence, form categorical classes of objects, label and enumerate natural and unnatural objects, fashion concepts of same/different, and display some comprehension of relative size. ${ }^{1}$

The foregoing indicates that the core abilities underlying metaphor - the ability to form mental representations of objects, integrate sensory and affective experiences, and form relational concepts - are widely distributed in the animal kingdom presumably because they have evolved to solve similar adaptive problems in the natural world or have emerged for complex social purposes that have driven the application of these abilities beyond their immediate Darwinian adaptations. For instance, sentinel behavior or the need to protect groups of conspecifics from predators, may have been part and parcel of the ability to categorize objects in the natural world, including conspecifics, and for doing so in a flexible manner using available information in the environment (Pepperberg, 1999; Seitz, 1999c). Such a "universal toolkit" or the evolution of domain-general abilities that are common across species - the ability to recognize objects, enumerate, navigate, and acquire knowledge (Hauser, 2000) - could form the basis of a species' ability to relate percepts, affects, and concepts across disparate domains of experience, i.e., create the conditions for the evolution of the ability to make metaphoric discriminations. Indeed, the ability to

\footnotetext{
${ }^{1}$ Physiognomic perception may extend to other species such as canines, at least according to anecdotal observation. At 14 months my Yorkshire Terrier began barking at two small stone lion statues leading to an entrance off a New York City sidewalk that he had encountered for the first time in our daily walks.
} 
combine simpler elements into more complex structures appears to be a fundamental capacity of biological systems (Marcus, 2004).

The developmental (i.e., ontogenetic), evolutionary (i.e., phylogenetic), and comparative evidence, thus, strongly suggest that perceptual, enactive, synesthetic, and physiognomic metaphoric abilities $\left(\mathrm{B}_{\mathrm{m}} \mathrm{T}\right)$ emerge very early in life, are biologically grounded in the ability to perceive likeness across certain domains, and may further lie at the core of creative thought. Only secondarily, as we have argued elsewhere (Seitz, 1997, 1998b; the philosophical basis for this view is presented in Goodman, 1976), is the ability to perceive likeness or nonliteral similarity "captured" in different symbol systems (e.g., gestures, words, pictures, numbers, music, dance notation, and so on). We review below the evidence for the bodily basis of these fundamental metaphoric abilities $\left(B_{m} T\right)$.

\section{Bodily basis of metaphor}

\subsection{Motor and nonmotor areas of the brain overlap}

The evidence for the bodily or "embodied" basis of early metaphor $\left(\mathrm{B}_{\mathrm{m}} \mathrm{T}\right)$ is fourfold. In the first instance, many of the so-called motor structures in the brain (e.g., cerebellum, basal ganglia, and supplementary motor, premotor and motor cortices) play a significant role in cognition (Seitz, 2000a). For example, the acquisition in children of two-word utterances during the second half of the second year of life is revealing. Roger Brown (1973) has documented that eleven types of sensorimotor experience are commonly manifested in infants' initial two-word combinations (see also Gardner, 1982). For instance, the relationship of agent performing an action is established during the sensorimotor period and then later "mapped" onto early language (e.g., "Johnny fall"). These eleven fundamental types of bodily kinesthetic knowledge include forms of reference (naming, recurrence, and nonexistence) as in the linguistic utterance, "There doggie;" various sets of relations (agent-action, agent-object, action-object, action-location, and object-location) as in the utterance, "Book table;" possessor and possession (e.g., "My ball"); object attribution (e.g., "Little story"); and the category of demonstrative entity in which object specifications are applied to objects (e.g., "That ball"). These abilities are made possible because brain areas for motor and cognitive functions overlap and are interdependent and in acquiring conceptual knowledge, the two are inextricably intertwined (Seitz, 2000a). Thus, enactive or "action metaphors" (Dent, 1984) in which a spinning top is likened to a dancing bear, emerge in early childhood presumably as a consequence of the acquisition of sensorimotor knowledge acquired during infancy.

\subsection{Mirror neurons}

A second piece of evidence is that there is a "mirror system" for both action execution and action recognition in the primate cortex suggesting the close association between intended movements carried out by an actor and observed movement performed by some viewer (Rizzolatti \& Arbib, 1998). This system is underwritten by "mirror neurons" in the premotor cortex that link externally observed events (i.e., manual signs and gestures, postural attitudes, facial expression, eye movements and gaze, vocal prosody, whole-body movements, and rhythm) to internally generated motor actions and establishes a coordinative relationship between gesture and intentional communication facilitating 
both the establishment of intentional mental states and the ability to "read" them in others (Gallese \& Goldman, 1998). This mirror system would enable primates to model external events in self-initiated motor behaviors (i.e., mimetic skills; Donald, 1991) and such a capacity would have enabled intentional (nonverbal) communication and eventually, speech. Ramachandran and Hubbard $(2001 \mathrm{a}, \mathrm{b})$ argue that this mirror system would underwrite "sensorimotor synesthesia" or the mimicking of auditory rhythm by rhythmic movements. Forms of motor-to-motor mappings ("synkinesia") would also be mediated by mirror neurons and underlie enactive metaphor.

Moreover, this mirror system is generative. It has been credibly argued that there is a general purpose sequencing module in the brain that parses (motor) action into its components, recombines these components, and these recombined components underlie both movement and speech (Corballis, 1999; Greenfield, 1991). Spoken language and movement (e.g., gesture) would draw on the same underlying brain systems. Since, it is assumed that gesture preceded speech in hominid evolution, it has been hypothesized that the evolutionary basis for vocal languages originates in gesture (Corballis, 2002; Hewes, 1973). Contemporary studies of signed languages tend to bear this out. For instance, sign language expresses metaphoric meaning through the modification of sign by way of the overlapping, blending, and substitution of signed gestures (Klima \& Bellugi, 1980; Sternberg, 1999). Ironic and metaphoric modes of communication are quite common in deaf communities including esthetic uses of sign as in deaf theater or sign poetry. Deaf children, too, develop the capacity to produce figurative sign language through use of pantomime, ritualized movement, sign modification, and by adding iconic, visuospatial detail (Marschark, Everhart, Martin, \& West, 1987). Similarly, research has shown that blind subjects are able to depict motion metaphorically in drawings and will draw, for example, curved spokes on paper to depict the movement of a wheel (Kennedy, 1997). Indeed, both deaf and blind subjects - whether children or adults - make use of enactive and perceptual metaphor in acquiring sign language, Braille or a spoken language (Winner et al., 1979).

\subsection{Cross-modal perception}

In the third instance, there is evidence for cross-modal perception in infants and very young children that arises from the close association between sensory, perceptual, and cognitive processes that are intimately tied to the body (e.g., Asch \& Nerlove, 1960; Spelke, 1981; Stern, 1985; Stern, Hofer, Haft, \& Dore, 1985; Wagner et al., 1981). For instance, a recent study demonstrated that 4-, 6-, and 8-month olds can discriminate visible, audible, and bimodal components of isolated syllables (Lewkowicz, 2000). In this study, both kinematic visual information and complex auditory information (e.g., vocal prosody) enhanced infants' responses to bimodal continuous utterances. Nonetheless, Lewkowicz (1994) claims that while very young infants appear to match intersensory stimuli based on equivalence of intensity, older infants and very young children match cross-modal stimuli based on complete multisensory integration that includes the concatenation of sensory, perceptual, and cognitive information. A similar view is proposed by Maurer (1997; Maurer \& Mondloch, 2005). She suggests that early cross-modal abilities are only apparent in early infancy - so-called "neonatal synesthesia"- based on cross-modal confusion and the lack of cortical specialization. True cross-modal transfer appears to kick in before the end of the first year or sooner and is based on connections among fully differentiated sensory areas. 


\subsection{Representation of the body schema}

Finally, there is a evidence for a distributed neural network, including the somatosensory cortex, limbic system, and cortical regions central to object- and selfrecognition, that form the neural basis for corporeal awareness, i.e., one's representation of one's body or "body schema" (Berlucchi \& Aglioti, 1997). One's sense of self and personal identity are constructed from one's body, namely, body-centered representations that are modulated over time by changes to one's body schema or plan (Seitz, 2000b). It is plausible that the ability to perceive and exploit early visual-affective and other sensoryaffective relations that rely on physiognomic characteristics - the outward appearance or character of an individual - is a result of (a) the emergence of this distributed neural network very early in development and, (b) evidence that this network forms the basis for bodily awareness. To be sure, it has been suggested that in understanding others a crossmodal representational system has evolved that binds self and other principally through behavioral imitation. Such a cognate system appears to be innate and allows even very young infants (e.g., 6-weeks old) to imitate others based on their own primitive body scheme (Gopnik, 1993; Meltzoff \& Moore, 2002).

We review below the evidence for the neuropsychological basis of these fundamental metaphoric abilities $\left(\mathrm{B}_{\mathrm{m}} \mathrm{T}\right)$.

\section{Neuropsychological evidence}

\subsection{Historical background}

Initial observations of the brain basis of basic metaphoric abilities $\left(\mathrm{B}_{\mathrm{m}} \mathrm{T}\right)$ indicated that intersensory correlations as well as physiognomic experiences derived from a primitive, amodal core in which perceptual, affective, and motoric aspects of experience were undifferentiated in early development (Werner, 1948, 1955, 1978; Werner \& Kaplan, 1963/ 1984). Werner termed these synesthetic associations "comparison metaphors." More recent studies have shown that these synesthetic associations are universal across languages and cultures and presumably innate (e.g., Asch, 1955; Brown, 1958; Dey, 1996; Gerrig \& Banaji, 1994; Osgood, 1960).

With regard to the brain basis of language abilities, Geschwind (1964) contended that language ability arose from cross-modal associations (i.e., the collation of vision, audition, and somesthesis-bodily sensations - in the association cortices) between nonlimbic modalities (e.g., visual-auditory, tactile-auditory) in the inferior posterior parietal cortex (i.e., angular gyrus). He hypothesized that grammar was an intramodal, i.e., auditory-auditory association. Although he argued that these cross-modal association areas were not fully myelinated until the end of the fourth year of life, recent evidence - reviewed below-suggests that these abilities emerge earlier in infancy.

\subsection{Synesthesia}

Marks and Bornstein (1987; Marks, 1996; Marks et al., 1987) allege that synesthetic metaphors (e.g., brightness-loudness and brightness-pitch relationships) are based on innate perceptual similarities whereas synesthetic metonymies (e.g., pitch-size and colortemperature relationships) are learned figurative relations. They argue that synesthesia or 
cross-modal perception (found in all normal individuals) provides the biological infrastructure for (synesthetic) metaphorical mappings, as these putative innate perceptual similarities are fixed and determinate as regards order, direction, and polarity. They contend that they represent primitive perceptual forms that are created by means of polysensory cortical neurons and integrated at cortical association areas. On the other hand, metonymies are associative equivalences based on learned experience and are more abstract and flexible; in extending them to nonsensory categories analogies are created. Thus, synesthetic associations are argued to be one of the core abilities underlying more advanced forms of metaphor and analogy.

The existence of heightened or "true" synesthesia in adults who experience unusually strong cross-modal associations (e.g., colored hearing and gustation, shaped audition and gustation, and textured and colored speech) is a relatively rare phenomenon, however (Cytowic, 1993, 1995, 2002a,b; Cytowic \& Wood, 1982a,b). Cytowic originally hypothesized that the hippocampus might be the site of cross-modal integration as it has been argued to be one of the major multimodal "convergence zones" in the cortex (e.g., Damasio \& Damasio, 1994; Fuster, 2003). There is no convincing evidence, however, that the primary function of the hippocampus is to integrate sensory information across disparate modalities. This may occur earlier in the processing stream extending from more downstream unimodal sensory cortices to upstream multimodal convergence zones. Several of the latter have been proposed for the major lobes of the cerebral cortex (Pandya \& Yeterian, 1990).

Cytowic also suggested that the anomalous cerebral dominance hypothesis proposed by Norman Geschwind may underlie such heightened synesthetic experiences. The anomalous dominance hypothesis asserts that hormonal influences on the developing brain (i.e., androgens) contribute to differences in the rate and extent of development of the right and left cerebral hemispheres relative to each other (Devinsky \& Schachter, 1997) and such a situation may affect connections among relevant brain areas in synesthesia. Ramachandran and Hubbard (2001a, b) and Cytowic (2002a, b), however, have more recently argued that such heightened synesthesia may be an X-linked mutation causing excessive propagation or faulty pruning of neural connections in early development. As a result, there is abnormal cross-wiring between, for example, areas of color (V4) and number (fusiform gyrus), between sound (auditory cortex) and motor areas (adjacent to Broca's area) or shape (inferior temporal), or between color (V4) and auditory maps in the superior temporal gyrus. Heightened affective reactions experienced during such states may be due to hyperactivation of limbic structures (e.g., amgydala, nucleus accumbens). Nonetheless, the underlying brain basis and unconscious origins of synesthesia in normal adults and children indicates the ubiquitousness of synesthetic associations in normal human populations.

\subsection{Multisensory and multimodal integration}

Multisensory or multimodal integration is the ability to access a shared representation through diverse sensory or brain pathways (Allen \& Bekoff, 1997). Research on the brain basis of multisensory integration indicates that the superior colliculus (SC), the anterior ectosylvian cortex, and the rostral portion of the lateral suprasylvian sulcus - structures located in the midbrain and frontal cortices - are comprised of multisensory neurons that facilitate multisensory integration of visual, auditory, somatosensory, and motor 
experiences in individuals (Stein \& Meredith, 1993; Stein, Laurienti, Wallace, \& Stanford, 2002; Stein, Stanford, Vaughan, and Wallace, 1999; Strehler, 1991). To be sure, it has been demonstrated that in the primate cortex the integration of vision, touch, proprioception, and motor feedback occurs in area 5 of the parietal cortex (i.e., intraparietal sulcus or IPS, a midbrain structure) and underlies representation of the body plan (Graziano, Cooke, \& Taylor, 2000). It thus may provide the neural basis of physiognomic metaphor $\left(B_{m} T\right)$ along with ventral visual areas of the cortex (V4 for facial perception) and the amygdala and related limbic areas for the perception of emotion (Kolb \& Whishaw, 2003).

Area V5 in the superior temporal sulcus (STS) appears to directly serve the detection of biological motion (Allison, Puce, \& McCarthy, 2000) and would seem to be a likely candidate for the neural basis of enactive metaphor in $\mathrm{B}_{\mathrm{m}} \mathrm{T}$ as well as auditory and rhythmic synchronicity (the latter mediated by the right posterior superior temporal gyrus; Liegeois-Chauvel, Peretz, Babai, Laguitton, \& Chauvel, 1998) in the human perception of music and cognate forms of cross-modal perception (Seitz, 2005). The STS appears to function as a convergence zone for integration of the dorsal "where' and the ventral "what" visual pathways as well as having important connections to the amygdala and orbitofrontal cortex (OFC) for the interpretation of stimuli. The inferior temporal cortex that houses memory for shape along with V4 (color) and V3 (dynamic form) in the occipital cortex (Zeki, 1999) would be appear to be likely candidates for the neural basis of perceptual metaphor in $\mathrm{B}_{\mathrm{m}} \mathrm{T}$.

The SC and an adjacent area in the cortex, the insula, appear to be implicated in the amalgamation of crossmodal information based on temporal correspondence whereas the inferior parietal sulcus (IPS) seems to be involved in the assemblage of cross-modal information based on spatial correspondence, particularly in the anterior region of the IPS or "AIP" (Heilman, Watson, \& Valenstein, 2003). Both of these and related structures would be probable candidates for synesthetic or cross-modal metaphor in $\mathrm{B}_{\mathrm{m}} \mathrm{T}$. To be sure, the STS has been posited to coordinate audiovisual information in speech whereas the insula and claustrum (CS), which are situated next to each other in the midbrain, appear to integrate the visual and tactile modalities. The rhinal cortex in the medial temporal region is proposed to be involved in the storage of multimodal representations, the claustrum in their cortical synchronization, especially cortical synchronization of sensory-specific cortices, and the SC playing a major role in the mediation of orienting and attentive behaviors (Calvert, 2001).

Indeed, Calvert (2001) maintains that both cortical and subcortical structures are involved in multisensory and multimodal integration at several heteromodal sites. The cortical sites include the STS, the IPS, and areas in the frontal and parietal lobes. She reasons that the frontal cortex is implicated in the initial learning of newly acquired multimodal associations whereas the STS appears to be involved in the subsequent learning of amodal representations. The IPS facilitates the multimodal integration of spatial coordinate information, as described above, as well as the integration of vision and touch.

The subcortical sites include the supergeniculate and medial pulvinar nuclei of the thalamus, which connect to the SC, and visual and auditory areas. On the other hand, the nucleus reticularis thalami (NRT) is responsive to polymodal cortices. When inhibited by these cortices it increases cortical arousal and potentiates the mesencephalic reticulating system (MRF) enabling the organism to be more responsive to novel and biologically significant stimuli - thus, enhancing the brain's ability to pick out relevant percepts based on these fundamental types of metaphoric processes described in $\mathrm{B}_{\mathrm{m}} \mathrm{T}$. 
On that score, Stein et al. $(1999,2002)$ contend that multisensory integration evolved in primates and others species to increase the probability of stimulus detection - particularly stimulus novelty and significance-heighten the ability of organisms to simultaneously monitor multiple environmental cues, and facilitated both speed and accuracy of identification as well as the ability to detect misinformation (Allen \& Bekoff, 1997). Creative thought in human primates, thus, would represent an exaptation or Darwinian preadaptation of these multimodal abilities that originally evolved for the servicing of more mundane activities essential to survival in other species. Ramachandran and Hubbard (2001b, 2005) have made similar claims for the evolutionary role of the angular gyrus in synesthetic metaphor. That is, the angular gyrus interleaves the spatial modalities (audition, vision, and touch) creating the preconditions for abstract thought - extracting correlated sensory information (e.g., sound, shape, and texture) to arrive at a more complex percept or abstract property - for instance, "jaggedness."

Thus, the above cortical and subcortical structures would provide the biological infrastructure for the integration of sensory, perceptual, affective, and motor information found in perceptual, enactive, physiognomic, and synesthetic metaphors $\left(B_{m} T\right)$. We now turn to some alternative views of early metaphor.

\section{Alternative views of early metaphor}

\section{1. "Primary" metaphor}

The foregoing description of four species of early metaphor and their emergence early in development is by no means the only perspective on early metaphor. For instance, Lakoff and Johnson (1999) allege that there are several hundred, universal metaphors that are acquired early in development. For example, "IMPORTANT IS BIG" "DIFFICULTIES ARE BURDENS," and "LINEAR SCALES ARE PATHS." These universal, conceptual, and linguistic metaphors ("primary" metaphor, Lakoff \& Johnson, 1999) are acquired early in development through a process of "conceptual mapping via neural connections" by means of "coactivation" (Lakoff \& Johnson, 1999, p. 57). That is, in the process of acquiring metaphoric relations, new conceptual mappings establish cellular changes in neurons because of changes at synaptic junctures of neurons, according to Lakoff and Johnson (1999). These changes are driven by what the child is exposed to in the environment based on a process of coactivation or experiential similarity or co-occurrence (Lakoff \& Johnson, 1980).

The difficulty with this view, however, is that it does not take into account the preliterate (evolutionary basis), prelinguistic (i.e., early development in children prior to the emergence of language), and extralinguistic (e.g., nonverbal, gestural) bases of metaphoric activity prior to the development of speech and language or the fact that these "primary" metaphors or cognate childhood utterances are not found in the speech or language of young children. Examples of the above "primary" metaphors would include, "Tomorrow is a big day," "She's weighed down by responsibilities," and "John's intelligence goes way beyond Bill's," respectively. In effect, these are actually later developing conceptual metaphors. Such "secondary" metaphors as "UNDERSTANDING IS GRASPING" as in "I've never been able to grasp transfinite numbers" or "HAPPY IS UP" as in "I'm feeling up today" are acquired conceptually later in development from-among other things - similarity encountered in the environment (e.g., noting the similarity between 
change and motion as in "My car has gone from bad to worse lately") or as a result of encountering correlations between domains as a result of their co-occurrence (e.g., experiencing "organization" and "physical structure" together as in "How do the pieces of this theory fit together?"). These are learned conceptual relations and are built upon prior category abilities and do not appear to index early metaphoric abilities that arise from early sensory, perceptual, affective, and cognitive abilities found in $\mathrm{B}_{\mathrm{m}} \mathrm{T}$.

\subsection{Other perspectives}

Other perspectives on early metaphor suggest that early metaphor gives rise to highly abstract metaphorical "blends" that abet creative discovery (i.e., "basic" metaphor, Fauconnier \& Turner, 1998, 2002) or suggest that early metaphor works outside awareness to structure our everyday folk conceptions (i.e., "implicit" metaphor, Vervaeke \& Kennedy, 1996) and may either cohere in a web of beliefs or result from experiential similarity or co-occurrence. Grady $(1998,1999)$ and Grady, Taub, and Morgan (1996) argue that complex metaphors are compound structures that are decomposable into simpler submappings or "primitives" and suggest that they may arise from some experiential basis or unspecified neurological structure. ${ }^{2}$ Of the former, they may originate in correlations between dimensions, or components, of experience. Nonetheless, no developmental evidence is provided to support these various notions of early metaphor. In a small corpus of adult-child language samples, Johnson (1997) suggests that adult language picks out the relevant dimensions for the child. Children's use of metaphor, however, emerges in domain-specific ways in development, is frequently spontaneous, and is often not reflective of adult categories (Winner, 1988, 1995). For instance, pretend action metaphors are the preponderance of metaphors in the third year, but by the fifth year perceptual metaphors dominate (Winner, 1988). Certainly, adults do not regularly attend to those features in their speech or actions such that children could acquire these abilities from adult feedback alone. Thus, it is difficult to see how a learning model could adequately account for the emergence of early metaphor.

\subsection{The conflation view of early metaphor}

Lakoff and Johnson (1999) and Johnson (1997), however, in several studies of the language corpora of young children, maintain that children's domains are "conflated" in early development. That is, they suggest that early metaphor emerges in the second of two stages. In the first or conflation stage, occurring ostensibly in infancy and through early childhood, connections between domains are completely interleaved and are experienced by the infant or child as one and the same. Under such a developmental scenario, metaphoric associations are not possible. In the second or differentiation stage, occurring in later childhood, metaphorical associations are established between a source (i.e., a sensorimotor experience) and a target (i.e., a nonsensorimotor experience). Thus, on their view, synesthetic and other kinds of metaphoric associations would not emerge until later childhood. They suggest that the domains being conflated are sensorimotor experiences

\footnotetext{
${ }^{2}$ Grady et al. (1996) and Grady (1998) address related problems with Lakoff and Johnson's formulation of complex metaphors as decomposable into primitives such as the notion of what counts as the experiential basis of a metaphor.
} 
and nonsensorimotor experiences and once these domains have been "coactivated" by way of cultural learning, neural connections develop between the domains such that there is a differentiation of the source and target enabling the emergence of conceptual metaphor.

There is an abundance of experimental evidence, however, that indicates that even very young infants are capable of perceiving perceptual, enactive, cross-modal, and other kinds of metaphoric similarity (e.g., Maurer, 1997; Maurer \& Mondloch, 2005; Meltzoff \& Borton, 1979; Lewkowicz, 1994, 2000; Starkey, Spelke, \& Gelman, 1983; Stern, 1985; Stern et al., 1985: Wagner et al., 1981). Indeed, early metaphoric abilities in the perceptual domains of color, shape, texture, and size; movement; and cross-sensory equivalence, are established in late infancy and physiognomic perception by the middle of the third year, at the latest. Innate factors play a significant, but not exclusive, role in the emergence of early metaphor. Hence, the available scientific evidence does not support a notion of a conflation stage in metaphoric development.

\subsection{Basic and conceptual metaphor emerge in two stages}

An alternative position is that metaphoric creation and understanding emerge in a twostage process in which inborn metaphorical associations give rise to later developing conceptual metaphor (Seitz, 1997). In the first stage, early metaphor is largely innately based and reliant on underlying brain and bodily mechanisms (see Section 3.5). These early or primary metaphoric associations would include the perceptual, enactive, cross-modal, and physiognomic realms that, we have argued, unmistakably emerge early in development $\left(\mathrm{B}_{\mathrm{m}} \mathrm{T}\right)$. Lakoff, Grady, and colleagues suggest that such early metaphor does not exist because ostensibly, (a) more time is needed in the infant or young child's life for such learning to occur (Lakoff \& Johnson, 1999), or (b) although more primitive metaphoric structures may in fact exist, they do not adequately specify how they enter into any theory of the development of more complex metaphor implicit in the $\mathrm{S}_{\mathrm{m}} \mathrm{T}$ (Grady, Taub, \& Morgan, 1996).

\section{Conclusions}

We believe that our $B_{m} T$ explains more about how people actually recognize or create basic or primary metaphoric associations across disparate domains of experience than does that $\mathrm{S}_{\mathrm{m}} \mathrm{T}$ partly because humans are "pre-wired" to make these linkages. These basic metaphoric equivalences include perceptual-perceptual, movement-movement, crossmodal (synesthetic), and perceptual-affective relations that we demonstrated are uniquely mapped onto brain "networks." We presented three major strands of scientific support for this view: preliterate (i.e., evolutionary), prelinguistic (i.e., developmental), and extralinguistic (i.e., neuropsychological and cognitive) evidence. These basis metaphoric relations generally operate outside of conscious awareness as compared to secondary or conceptual metaphor, which, we propose, is typically an amalgam of both conscious and unconscious processes.

$\mathrm{S}_{\mathrm{m}} \mathrm{T}$ has typically ignored these linkages instead addressing problems with how people process, recognize, produce or understand secondary or conceptual metaphor. We believe our theory has the advantages of elegance and simplicity over the $\mathrm{S}_{\mathrm{m}} \mathrm{T}$ in better explaining the everyday and garden-variety metaphoric experiences of children and adults. 


\section{Acknowledgments}

The author would like to thank Professor Harry Beilin of The Graduate Center/City University of New York (CUNY), Professor John Kennedy of the University of Toronto, Professor Elkhonon Goldberg of NYU School of Medicine, and several anonymous reviewers for their helpful comments and suggestions on earlier drafts of this manuscript.

\section{References}

Allen, C., \& Bekoff, M. (1997). Species of mind: The philosophy and biology of cognitive ethology. Cambridge, MA: MIT Press.

Allen, R., \& Reber, A. S. (1998). Unconscious intelligence. In W. Bechtel, \& G. Graham (Eds.), A companion to cognitive science (pp. 314-323). Oxford: Blackwell.

Allison, T., Puce, A., \& McCarthy, G. (2000). Social perception from visual cues: Role of the STS region. Trends in Cognitive Sciences, 4(7), 267-278.

Anati, E. (1994). World rock art: The primordial language. Valcamonica, Italy: Edizioni Del Centro.

Asch, S. E. (1955). On the use of metaphor in the description of persons. In H. Werner (Ed.), On expressive language (pp. 29-38). Worcester, MA: Clark University Press.

Asch, S. E., \& Nerlove, H. (1960). The development of double-function terms in children. In B. Kaplan, \& S. Wapner (Eds.), Perspectives in psychological theory (pp. 47-60). New York: International Universities Press.

Baron-Cohen, S., Leslie, A. M., \& Frith, U. (1985). Does the autistic child have a "theory of mind"? Cognition, $21,37-46$.

Berlucchi, G., \& Aglioti, S. (1997). The body in the brain: Neural bases of corporeal awareness. Trends in Neurosciences, 20(12), 560-564.

Bowers, K. S., Regehr, G., Balthazard, C., \& Parker, K. (1990). Intuition in the context of discovery. Cognitive Psychology, 22(1), 72-110.

Bownds, M. D. (1999). The biology of mind: Origins and structures of mind, brain, and consciousness. Bethesda, MD: Fitzgerald Science Press.

Brothers, L. (1989). A biological perspective on empathy. American Journal of Psychiatry, 146(1), 10-19.

Brown, D. E. (1991). Human universals. Boston, MA: McGraw-Hill.

Brown, D. E. (1999). Human universals. In R. A. Wilson, \& F. C. Keil (Eds.), The MIT encyclopedia of the cognitive sciences (pp. 382-384). Cambridge, MA: MIT Press.

Brown, D. E. (2000). Human universals and their implications. In N. Roughley (Ed.), Being human: Anthropological universality and particularity in transdisciplinary perspectives (pp. 156-174). Berlin: Walter de Gruyter.

Brown, R. (1973). A first language: The early stages. Cambridge, MA: Harvard University Press.

Brown, R. W. (1958). Is a boulder sweet or sour? Contemporary Psychology, 3, 113-115.

Calvert, G. A. (2001). Crossmodal processing in the human brain: Insights from functional neuroimaging studies. Cerebral Cortex, 11, 1110-1123.

Cicone, M., Gardner, H., \& Winner, E. (1981). Understanding the psychology in psychological metaphors. Journal of Child Language, 8(1), 213-216.

Corballis, M. (1999). The gestural origins of language. American Scientist, 87(2), 138-145.

Corballis, M. (2002). From hand to mouth: The origins of language. Princeton, NJ: Princeton University Press.

Cytowic, R. E. (1993). The man who tasted shapes. New York: Putnam.

Cytowic, R. E. (1995). Synesthesia: Phenomenology and neuropsychology. Psyche, 2(10) http://psyche.cs.monash.edu.au/v2/psyche-2-10-cytowic.html.

Cytowic, R. E. (2002a). Touching tastes, seeing smells - and shaking up brain science. Cerebrum, 4(3), 7-26.

Cytowic, R. E. (2002b). Synesthesia: A union of the senses (2nd ed.). Cambridge, MA: MIT Press.

Cytowic, R. E., \& Wood, F. B. (1982a). Synesthesia: I. A review of major theories and their brain basis. Brain and Cognition, 1, 23-35.

Cytowic, R. E., \& Wood, F. B. (1982b). Synesthesia: II. Psychophysical relations in the synesthesia of geometrically shaped taste and colored hearing. Brain and Cognition, 1, 36-49. 
Damasio, A. R., \& Damasio, H. (1994). Cortical systems for retrieval of concrete knowledge: The convergence zone framework. In C. Koch, \& J. L. Davis (Eds.), Large-scale neuronal theories of the brain (pp. 61-74). Cambridge, MA: MIT Press.

Dent, C. H. (1984). The developmental importance of motion information in perceiving and describing metaphoric similarity. Child Development, 55, 1607-1613.

Dey, S. (1996). Synaesthesia and synaesthetic metaphors. Psyche, 2(32) http://psyche.cs.monash.edu.au/v2/ psyche-2-32-day.html.

Devinsky, O., \& Schachter, S. C. (1997). Norman Geschwind: Selected papers on language, epilepsy, and behavior. Boston: Butterworth-Heinemann.

Donald, M. (1991). Origins of the modern mind: Three stages in the evolution of culture and cognition. Cambridge, MA: Harvard University Press.

Fauconnier, G., \& Turner, M. (1998). Principles of conceptual integration. In J. P. Koenig (Ed.), Discourse and cognition: Bridging the gap (pp. 269-283). Stanford, CA: CSLI Publications.

Fauconnier, G., \& Turner, M. (2002). The way we think: Conceptual blending and the mind's hidden complexities. New York: Basic Books.

Fodor, J. A. (1983). The modularity of mind. Cambridge, MA: MIT Press.

Fuster, J. M. (2003). Cortex and mind: Unifying cognition. Oxford: Oxford University Press.

Gallese, V., \& Goldman, A. (1998). Mirror neurons and the simulation theory of mind-reading. Trends in Cognitive Sciences, 12(2), 493-501.

Gardner, H. (1974). Metaphors and modalities: How children project polar adjectives onto diverse domains. Child Development, 45, 84-91.

Gardner, H. (1982). Developmental psychology: An introduction (2nd ed.). Boston: Little, Brown \& Co.

Gentner, D., Holyoak, K. J., \& Kokinov, B. K. (Eds.). (2001). The analogical mind: Perspectives from cognitive science. Cambridge MA: MIT Press.

Gentner, D., \& Markman, A. B. (1997). Structure mapping in analogy and similarity. American Psychologist, 53(1), 45-56.

Gerrig, R. J., \& Banaji, M. R. (1994). Language and thought. In R. J. Sternberg (Ed.), Thinking and problem solving (pp. 233-261). San Diego: Academic Press.

Geschwind, N. (1964). The development of the brain and the evolution of language. In C. I. J. M. Stuart (Ed.), Monograph series on languages and linguistics, Vol. 17 (pp. 155-169). Washington, DC: Georgetown University Press.

Glucksberg, S. (2003). The psycholinguistics of metaphor. Trends in Cognitive Sciences, 7(2), 92-96.

Glucksberg, S., \& Keysar, B. (1993). How metaphors work. In A. Ortony (Ed.), Metaphor and thought (2nd ed., pp. 401-424). Cambridge, UK: Cambridge University Press.

Gombrich, E. H. (1963). Meditations on a hobby horse and other essays on the theory of art. London: Phaidon.

Goodman, N. (1976). Languages of art: An approach to a theory of symbols. Indianapolis: Hackett.

Gopnik, A. (1993). Psychopsychology. Consciousness and Cognition, 2, 264-280.

Gould, S. J. (1977). Ontogeny and phylogeny. Cambridge, MA: Harvard University Press.

Grady, J. (1998). The "conduit metaphor" revisited: A reassessment of metaphors for communication. In J. P. Koenig (Ed.), Discourse and cognition: Bridging the gap (pp. 205-218). Stanford, CA: CSLI Publications.

Grady, J. \& Johnson, C. (1999). Converging evidence for the notions of subscene and primary scene. Unpublished manuscript.

Grady, J., Taub, S., \& Morgan, P. (1996). Primitive and compound metaphors. In A. E. Goldberg (Ed.), Conceptual structure, discourse and language (pp. 177-187). Stanford, CA: CSLI Publications.

Graziano, M. S. A., Cooke, D. F., \& Taylor, C. S. R. (2000). Coding the location of the arm by sight. Science, 290, 1782-1786.

Greenfield, P. M. (1991). Language, tools, and brain: The ontogeny and phylogeny of hierarchically organized sequential behavior. Behavioral and Brain Sciences, 14, 531-595.

Gregory, R. C. (Ed.). (1998). The Oxford companion to the mind. Oxford: Oxford University Press.

Hauser, M. D. (2000). Wild minds: What animals really think. New York: Henry Holt \& Co.

Heilman, K. M., Watson, R., \& Valenstein, E. (2003). Neglect and related disorders. In K. M. Heilman, \& E. Valenstein (Eds.), Clinical neuropsychology (4th ed., pp. 296-346). Oxford: Oxford University Press.

Hewes, G. W. (1973). Primate communication and the gestural origin of language. Current Anthropology, 14(1-2), $5-24$. 
Johnson, C. (1997). Metaphor vs. conflation in the acquisition of polysemy: The case of 'see'. In M. K. Hiraga, C. Sinha, \& S. Wilcox (Eds.), Cultural, typological and psychological perspectives in cognitive linguistics. London: John Benjambins.

Kanner, L. (1946). Irrelevant and metaphorical language in early infantile autism. American Journal of Psychiatry, 103, 242-246.

Kennedy, J. M. (1997). How the blind draw. Scientific American, 276(1), 76-81.

Kihlstrom, J. F. (1987). The cognitive unconscious. Science, 237, 1445-1452.

Kihlstrom, J. F. (2002). The unconscious. In V. S. Ramachandran (Ed.), Encyclopedia of the human brain, Vol. 4 (pp. 635-646). San Diego: Academic Press.

Klima, E. S., \& Bellugi, U. (1980). Wit and poetry in American sign language. In W. C. Stokoe (Ed.), Sign and culture (pp. 105-132). Silver Spring, MD: Linstok Press.

Koch, C. (2004). The quest for consciousness: A neurobiological approach. Englewood, CO: Roberts \& Company.

Kogan, N., Connor, K., Gross, A., \& Fava, D. (1980). Understanding visual metaphor: Developmental and individual differences. Monographs of the Society for Research in Child Development, 45(1, serial no. 183).

Kolb, B., \& Whishaw, I. Q. (2003). Fundamentals of human neuropsychology (5th ed.). New York: Worth.

Lakoff, G. (1993). The contemporary theory of metaphor. In A. Ortony (Ed.), Metaphor and thought (2nd ed., pp. 202-251). Cambridge, UK: Cambridge University Press.

Lakoff, G., \& Johnson, M. (1980). Metaphors we live by. Chicago: University of Chicago Press.

Lakoff, G., \& Johnson, M. (1999). Philosophy in the flesh: The embodied mind and its challenge to Western thought. New York: Basic Books.

Liegeois-Chauvel, C., Peretz, I., Babai, M., Laguitton, V., \& Chauvel, P. (1998). Contribution of different cortical areas in the temporal lobes to music processing. Brain, 121(10), 1853-1867.

Leslie, A. M., \& Frith, U. (1988). Autistic children's understanding of seeing, knowing and believing. British Journal of Developmental Psychology, 6, 315-324.

Lewkowicz, D. J. (1994). Development of intersensory perception in human infants. In D. J. Lewkowicz, \& R. Lickliter (Eds.), The development of intersensory perception: Comparative perspectives (pp. 165-203). Hillsdale, NJ: Erlbaum.

Lewkowicz, D. J. (2000). Infants' perception of the audible, visible, and bimodal characteristics of multimodal syllables. Child Development, 71(5), 1241-1257.

MacWhinney, B. (2002). The gradual emergence of language. In T. Givon, \& B. F. Malle (Eds.), The evolution of language out of pre-language (pp. 85-106). Amsterdam: J. Benjamins.

Marcus, G. (2004). The birth of the mind: How a tiny number of genes creates the complexities of human thought. New York: Basic Books.

Marks, L. E. (1996). On perceptual metaphors. Metaphor and Symbolic Activity, 11(1), 39-66.

Marks, L. E., \& Bornstein, M. H. (1987). Sensory similarities: Classes, characteristics, and cognitive consequences. In R. E. Haskell (Ed.), Symbolic structures: The psychology of metaphoric transformation (pp. 49-65). Norwood, NJ: Ablex.

Marks, L. E., Hammeal, R. J., \& Bornstein, M. H. (1987). Perceiving similarity and comprehending metaphor. Monographs of the Society for Research in Child Development, 52(1, serial no. 215).

Marschark, M., Everhart, V. S., Martin, J., \& West, S. A. (1987). Identifying linguistic creativity in deaf and hearing children. Metaphor and Symbolic Activity, 2(4), 281-306.

Maurer, D. (1997). Neonatal synesthesia: Implications for the processing of speech and faces. In S. Baron-Cohen, \& J. E. Harrison (Eds.), Synaesthesia: Classic and contemporary readings (pp. 224-242). Oxford: Blackwell.

Maurer, D., \& Mondloch, C. J. (2005). Neonatal synesthesia: A reevaluation. In L. C. Robertson, \& N. Sagiv (Eds.), Synesthesia: Perspectives from cognitive neuroscience (pp. 193-213). Oxford: Oxford University Press.

Meltzoff, A. N., \& Borton, R. W. (1979). Intermodal matching by human neonates. Nature, 282, $403-404$.

Meltzoff, A. N., \& Moore, M. K. (2002). Imitation, memory, and the representation of persons. Infant Behavior and Development: An International and Multidisciplinary Journal, 25(1), 39-61.

McNeill, D. (1992). Hand and mind: What gestures reveal about thought. Chicago: The University of Chicago Press.

Mithen, S. (1996). The prehistory of the mind: The cognitive origins of art, religion, and science. London: Thames and Hudson.

Osgood, C. E. (1960). The cross-cultural generality of visual-verbal synesthetic tendencies. Behavioral Science, 5, 146-169. 
Pandya, D. N., \& Yeterian, E. H. (1990). Architecture and connections of cerebral cortex: Implications for brain evolution and function. In A. B. Scheibel, \& A. F. Wechsler (Eds.), Neurobiology of higher cognitive function (pp. 53-84). New York: Guilford Press.

Pepperberg, I. M. (1999). The Alex studies: Cognitive and communicative abilities of grey parrots. Cambridge, MA: Harvard University Press.

Quinn, N. (1991). The cultural basis of metaphor. In J. W. Fernandez (Ed.), Beyond metaphor: The theory of tropes in anthropology (pp. 56-93). Stanford: Stanford University Press.

Ramachandran, V. S., \& Hubbard, E. M. (2001a). Synaethesia-A window into perception, thought, and language. Journal of Consciousness Studies, 8(12), 3-34.

Ramachandran, V. S., \& Hubbard, E. M. (2001b). Psychophysical investigations into the neural basis of synaesthesia. Proceedings of the Royal Society of London B, 268, 979-983.

Ramachandran, V. S., \& Hubbard, E. M. (2005). The emergence of the human mind: Some clues from synesthesia. In L. C. Robertson, \& N. Sagiv (Eds.), Synesthesia: Perspectives from cognitive neuroscience (pp. 147-190). Oxford: Oxford University Press.

Rizzolatti, G., \& Arbib, M. A. (1998). Language within our grasp. Trends in Neurosciences, 21(5), 188-194.

Searle, J. (2005). Consciousness: What we still don't know. The New York Review of Books, 42(1), 36-39.

Seitz, J. A. (1992). [Review of emotion concepts]. Metaphor and Symbolic Activity, 7(2), 99-101.

Seitz, J. A. (1996). Social deficits in autistic children: The role of emotion and empathy. Unpublished manuscript, Department of Political Science \& Psychology, York College, City University of New York (CUNY).

Seitz, J. A. (1997). The development of metaphoric understanding: Implications for a theory of creativity. Creativity Research Journal, 10(4), 347-353.

Seitz, J. A. (1998a). Metaphor, symbolic play, and logical thought in early childhood. Genetic, Social, and General Psychology Monographs, 123(4), 373-391.

Seitz, J. A. (1998b). Nonverbal metaphor: A review of theories and evidence. Genetic, Social, and General Psychology Monographs, 124(1), 121-143.

Seitz, J. A. (1999a). The bodily basis of thought. Paper presented at the 29th annual symposium of the Jean Piaget Society, Mexico City, Mexico. [On-line]. Available: http://www.york.cuny.edu/ seitz/PiagetTalk.html

Seitz, J. A. (1999b). Political science and creativity. In M. A. Runco, \& S. Pritzker (Eds.), The encyclopedia of creativity (pp. 417-421). San Diego: Academic Press.

Seitz, J. A. (Ed.). (1999c). Selfish sentinels in cooperative mammals (Letter to the editor) [Available:]. Science, 284(5420), 1640-1644 http://www.york.cuny.edu/ ^seitz/working.htm.

Seitz, J. A. (2000a). The bodily basis of thought. New Ideas in Psychology: An International Journal of Innovative Theory in Psychology, 18(1), 23-40.

Seitz, J. A. (2000b). The embodied self. Paper presented at the 30th annual symposium of the Jean Piaget Society, Montreal, Canada. [On-line]. Available: http://www.york.cuny.edu/ seitz/montreal.htm

Seitz, J. A. (2000c). Embodied cognition. Paper presented at the annual meeting of the American Psychological Society, Miami, FL.

Seitz, J. A. (2001a). Mind, musical improvisation, and the body: The microgenesis of jazz improvisation. In J. Seitz (Chair), Adaptive and maladaptive forms of embodied cognition. Paper presented at the 31st annual symposium of the Jean Piaget Society, Berkeley, CA. [On-line]. Available: http://www.york.cuny.edu/ seitz/ body.htm

Seitz, J. A. (2001b). A cognitive-perceptual analysis of projective tests used with children. Perceptual and Motor Skills, 93, 505-522.

Seitz, J. A. (2002). Mind, dance, and pedagogy. Journal of Aesthetic Education, 36(4), 37-42.

Seitz, J. A. (2003a). A communitarian approach to creativity. Mind, Culture, and Activity: An International Journal, 10(3), 243-247.

Seitz, J. A. (2003b). The political economy of creativity. Creativity Research Journal, 15(4), 385-392.

Seitz, J. A. (2005). Dalcroze, the body, movement, and musicality. Psychology of Music, 33(4), 419-435.

Seitz, J. A., \& Beilin, H. (1987). The development of comprehension of physiognomic metaphor in photographs. British Journal of Developmental Psychology, 5, 321-331.

Sober, E., \& Wilson, D. S. (1997). Unto others: The evolution and psychology of unselfish behavior. Cambridge, MA: Harvard University Press.

Spelke, E. S. (1981). The infant's acquisition of knowledge of bimodally specified events. Journal of Experimental Child Psychology, 31, 279-299.

Squire, L. R., \& Kandel, E. R. (1998). Memory: From mind to molecules. New York: W. H. Freeman. 
Starkey, P., Spelke, E. S., \& Gelman, R. (1983). Detection of intermodal numerical correspondences by human infants. Science, 222, 179-181.

Stein, B. E., Laurienti, P. J., Wallace, M. T., \& Stanford, T. R. (2002). Multisensory integration. In V. S. Ramachandran (Ed.), Encyclopedia of the human brain, Vol. 3 (pp. 227-241). San Diego: Academic Press.

Stein, B. E., \& Meredith, M. A. (1993). The merging of the senses. Cambridge, MA: MIT Press.

Stein, B. E., Stanford, T. R., Vaughan, J. W., \& Wallace, M. T. (1999). Multisensory integration. In R. A. Wilson, \& F. C. Keil (Eds.), The MIT encyclopedia of the cognitive sciences (pp. 574-575). Cambridge, MA: MIT Press.

Stern, D. N. (1985). The interpersonal world of the infant: A view from psychoanalysis and developmental psychology. New York: Basic Books.

Stern, D. N., Hofer, L., Haft, W., \& Dore, J. (1985). Affect attunement: The sharing of feeling states between mother and infant by means of inter-modal fluency. In T. Field, \& N. Fox (Eds.), Social perception in infants. Norwood, NJ: Ablex.

Sternberg, M. L. A. (1999). The American sign language dictionary [CD-ROM]. Seattle: Multimedia 2000.

Strehler, B. L. (1991). Where is the self? A neuroanatomical theory of consciousness. Synapse, 7, 44-91.

Tomasello, M., \& Call, J. (1997). Primate cognition. Oxford: Oxford University Press.

Vialou, D. (1996). Prehistoric art and civilization. New York: Harry N. Abrams.

Vervaeke, J., \& Kennedy, J. M. (1996). Metaphors in language and thought: Falsification and multiple meanings. Metaphor and Symbolic Activity, 11(4), 273-284.

Wagner, S., Winner, E., Cicchetti, D., \& Gardner, H. (1981). "Metaphorical" mapping in human infants. Child Development, 52, 728-731.

Wallas, G. (1926). The art of thought. London: Butler \& Tanner.

Werner, H. (1948). Comparative psychology of mental development. New York: International Universities Press.

Werner, H. (Ed.). (1955). On expressive language. Worcester, MA: Clark University Press.

Werner, H. (1978). Unity of the senses. In S. S. Barten, \& M. B. Franklin (Eds.), Developmental processes: Heinz Werner's selected writings (pp. 153-167). New York: International Universities Press.

Werner, H., \& Kaplan, B. (1963/1984). Symbol formation: An organismic-developmental approach to the psychology of language. Hillsdale, NJ: Erlbaum (Original work published 1963).

Winner, E. (1988). The point of words: Children's understanding of metaphor and irony. Cambridge, MA: Harvard University Press.

Winner, E. (1995). Introduction. 10th anniversary special issue: Developmental perspectives on metaphor. Metaphor and Symbolic Activity, 10(4), 247-253.

Winner, E., McCarthy, M., \& Gardner, H. (1980). The ontogenesis of metaphor. In R. P. Honeck, \& R. R. Hoffman (Eds.), Cognition and figurative language (pp. 341-361). Hillsdale, NJ: Erlbaum.

Winner, E., McCarthy, M., Kleinman, S., \& Gardner, H. (1979). First metaphors. In D. Wolf, \& H. Gardner (Eds.), Early symbolization: New directions for child development, Vol. 3 (pp. 29-41). San Francisco: JosseyBass.

Zeki, P. (1999). Inner visions: An exploration of art and the brain. Oxford: Oxford University Press. 